Research Paper

\title{
c-Myb and its Effector COX-2 as an Indicator Associated with Prognosis and Therapeutic Outcome in Colorectal Cancer
}

\author{
Ruting Xie ${ }^{1^{*}}$, Yongzhi Yang ${ }^{2,3^{*}}$, Huizhen Zhang ${ }^{4}, \mathrm{Hu}_{\mathrm{Liu}}{ }^{1}$, Jing Guo ${ }^{1}$, Huanlong Qin 5 , Yanlei Ma ${ }^{2,3}{ }^{凶}$, Ajay \\ Goel ${ }^{6 \bowtie}$, Xinxiang Li2,3凶 , Qing Wei ${ }^{1 凶}$ \\ 1. Department of Pathology, Shanghai Tenth People's Hospital, Tongji University, Shanghai200072, P.R. China \\ 2. Department of Colorectal Surgery, Fudan University Shanghai Cancer Center, Shanghai, 200032, China \\ 3. Department of Oncology, Shanghai Medical College, Fudan University, Shanghai, 200032, China \\ 4. Department of Pathology, Shanghai Sixth People's Hospital, Shanghai Jiaotong University, Shanghai200233, P.R. China. \\ 5. Department of Surgery, Shanghai Tenth People's Hospital Affiliated with Tongii University, Shanghai 200072, P. R. China \\ 6. Center for Translational Epigenomics and Oncology, Baylor Scott \& White Research Institute and Charles A Sammons Cancer Center, Baylor University \\ Medical Center, Dallas, TX, United States. \\ *Yongzhi Yang and Ruting Xie contributed equally to this work \\ $\square$ Corresponding authors: Qing Wei, Department of Pathology, Shanghai Tenth People's Hospital, Tongji University, 301 Yanchang Road, Shanghai 200072, P. \\ R. China. wei_qing1971@163.com (Qing Wei); Xinxiang Li, MD, PhD, Department of Colorectal Surgery, Fudan University Shanghai Cancer Center; Department \\ of Oncology, Shanghai Medical College, Fudan University, 270 Dong'an Road, Shanghai, 200032, China, Tel.: +86 21 64175590; Fax: +86 21 54175590, E-mail: \\ li_xinxiang@hotmail.com. Ajay Goel, Center for Gastrointestinal Research, Center for Epigenetics, Cancer Prevention and Cancer Genomics, Baylor Scott \&White \\ Research Institute and Charles A. Sammons Cancer Center, 3410 Worth Street, Suite 610, Dallas, TX 75246, USA. Tel: 214-820-2603; Fax: 214-818-9292. Email: \\ Ajay.Goel@BSWHealth.org. Yanlei Ma, Department of Colorectal Surgery, Fudan University Shanghai Cancer Center; Department of Oncology, Shanghai \\ Medical College, Fudan University, 270 Dong'an Road, Shanghai, 200032, China, Tel.: +86 21 64175590; Fax: +86 21 54175590, E-mail: yanleima@live.cn.
}

(c) Ivyspring International Publisher. This is an open access article distributed under the terms of the Creative Commons Attribution (CC BY-NC) license (https://creativecommons.org/licenses/by-nc/4.0/). See http://ivyspring.com/terms for full terms and conditions.

Received: 2018.05.15; Accepted: 2019.01.04; Published: 2019.02.28

\begin{abstract}
Background: One of our previous studies have demonstrated that the cancer suppressor miR-150 regulated the progression of colorectal cancer (CRC) by down-regulating v-myb avian myeloblastosis viral oncogene homolog (c-Myb). The purpose of present study was to evaluate the prognostic value of the expression of c-Myb and its effector, prostaglandin-endoperoxide synthase 2 (COX-2) in patients with CRC.

Methods: We used tissue microarrays (containing 202 CRC tissues and matched adjacent normal tissues) and conducted immunohistochemical analysis and western blotting analysis (containing 3 CRC tissues and matched adjacent normal tissues) to detect the expression of c-Myb and COX-2.

Results: Compared with the adjacent nontumorous tissues, both the expression levels of c-Myb and COX-2 were higher in the cancer tissues. A statistically significant correlation was found between the expression of c-Myb and COX-2. Elevated c-Myb and COX-2 were associated with more advanced tumor invasion and poorer overall survival by univariate analysis. Higher expression levels of both c-Myb and COX-2 were significantly associated with shorter overall survival for stage II and stage III patients with 5-Fu based chemotherapy. Multivariate analysis identified the lymph node involvement, distant metastatic spread and the elevated c-Myb and COX-2 as independent factors of poor prognosis for CRC.

Conclusions: In conclusion, the overexpression of both c-Myb and COX-2 would be of prognostic screening value in patients with CRC.
\end{abstract}

Key words: c-Myb; COX-2; colorectal cancer; prognosis

\section{Introduction}

Colorectal cancer (CRC) ranks among the second leading cause of cancer-related mortality in the United States and remains the fourth most common cause of cancer deaths worldwide[1]. The burden of
CRC in China is also a severe public health issue. In 2015, there were 191 thousand CRC deaths cases according to the data from the National Central Cancer Registry of China [2]. Although the 
improvements in early screening such as guaiac fecal occult blood tests (gFOBT), fecal immunochemical test (FIT) and fibercoloscope ameliorate therapeutics management of patients with early stage of this disease, the five-year overall survival rates of individuals with advanced and metastatic stages remain bleak due to the lack of useful and clinicallyactionable tumor biomarkers for the prognosis monitoring [3-5]. To date, only serum carcinoembryonic antigen (CEA) measurement is recommend for the over-all post-surgical surveillance of early recurrence in CRC. However, there is no universal standard for the frequency of CEA testing and its high false-positive rate limits its monitoring value. These data highlight the critical and imperative need for identification of effective and sensitive biomarkers for assisting surgeons in developing a therapeutics.

Recent researches emphasized a integrative strategy for molecular subtyping[6], but only few tissue biomarkers such as microsatellite instability (MSI) status, CpG island methylator phenotype (CIMP) status, RAS mutation, and BRAF mutation are applied in clinical-outcome judgement of CRC. Our previous studies have demonstrated that a tumorsuppressive miRNA, miR-150, regulates the tumorigenesis and progression of CRC by post-transcriptionally reducing the expression level of nuclear protooncogene, v-myb avian myeloblastosis viral oncogene homolog (c-Myb) in cultured cells and animal models[7,8]. As one of the important transcription factors, c-Myb is frequently elevated in a number of carcinomas including acute myelogenous leukemia $[9,10]$, salivary adenoid cystic carcinoma[11], breast cancer[12], and CRC[9]. This gene participates in a wide variety of biological life processes including cell proliferation, cell cycle progression, and apoptosis, indicating that the malignancy maintenance of CRC cells is c-Myb dependent[13-15]. Moreover, recent studies discovered the mediation of chronic inflammatory reaction by c-Myb in the gut[13-15]. These findings help us understand why antiinflammatory therapy is useful in the CRC treatment. Meanwhile, it also reminds us to determine the prognostic value of c-Myb and Inflammatory markers in the post-surgical surveillance of CRC.

As one of the downstream effectors of $\mathrm{c}-\mathrm{Myb}$, prostaglandin-endoperoxide synthase 2 (COX-2), is a consistent hallmark feature of gut inflammation and CRC [19]. Overexpression of COX-2 has been confirmed to play an important role in epithelialmesenchymal transition (EMT) and lymph node metastasis[20]. However, the clinicopathological relevance of upregulated expression levels of c-Myb in combination with COX-2, in determining the prognosis of CRC patients has not yet been studied.
The aim of present study was to determine the expression levels of $\mathrm{c}-\mathrm{Myb}$ and COX-2 in sporadic $\mathrm{CRC}$, and decipher their prognostic usefulness in this malignancy.

\section{Materials and Methods}

\section{Patient specimens and clinicopathological data collection}

Matched normal and cancerous colorectal tissues from sporadic CRC patients were obtained from 202 patients (Han Chinese) who received colorectal surgery at the Sixth People's Hospital affiliated with Shanghai Jiao Tong University, Shanghai, China, between January 2004 and October 2007. The study was approved by the committee from the Sixth People's Hospital affiliated with Shanghai Jiao Tong University. Written informed consent was obtained from the patients, in accordance with the institutional guidelines. The methods were performed in accordance with the approved guidelines. We excluded cases that were diagnosed as familial adenomatous polyposis or with colitis-associated CRC. The endpoints of interest was overall survival. The deadline of follow-up was 1, April 2011 for all CRC cases. Survivors among the cases at last contact were considered as censored for following overall survival analysis. All the patients' clinicopathological data including age, gender, tumor location, differentiation, tumor size, depth of invasion, lymph node involvement, distant metastatic spread, tumor node metastasis (TNM) stage, 5-fluorouracil (Fu)-based chemotherapy, KI-67 expression, P53 status, and CEA status were obtained from the electronic medical records of the Sixth People's Hospital affiliated with Shanghai Jiao Tong University. The summary of clinicopathological data was demonstrated in table 1.

\section{Tissue microarray construction}

Formalin-fixed and paraffin-embedded tumor blocks were collected from the archives of the pathological departments at the Sixth People's Hospital affiliated with Shanghai Jiao Tong University. After marked on the hematoxylin and eosin slide, the most representative tumor areas of the paraffin blocks were chosen and 2.0-mm dimension tissue cores were arranged in tissue microarray (TMA) blocks. Then, $4.0 \mu \mathrm{m}$ TMA sections were cut for immunohistochemistry (IHC) analysis.

\section{Immunohistochemistry and staining evaluation}

The sections were dried for $2 \mathrm{~h}$ at the $65^{\circ} \mathrm{C}$ hot air oven, de-waxed by dimethylbenzene and rehydrated in gradient alcohol. Antigen was retrieved in 
citric acid buffer( $\mathrm{PH}$ 6.0) with a pressure antigen retrieval procedure for $5 \mathrm{~min}$. After washing the slides by PBS buffer( $\mathrm{PH}$ 7.4), endogenous peroxidase activity was interdicted by incubating the sections for $10 \mathrm{~min}$ in endogenous peroxidase blocking solution at room temperature. Non-specific background was blocking by incubating with normal nonimmune goat serum at room temperature for $30 \mathrm{~min}$. The sections were then incubated with following primary antibodies: anti-c-Myb (Rabbit polyclonal, 1:400, Abcam, Cambridge, United Kingdom, ab117635) and anti-COX-2 (Rabbit polyclonal, 1:200, Abcam, Cambridge, United Kingdom, ab15191). The slices incubated with PBS buffer (PH7.4) only were used as negative control. After washing with the PBS buffer (PH7.4), the tissue sections were detected and visualized with the Polink-1 HRP DAB detection system (ZSGB-BIO, Beijing, China). Then the reaction was stopped under running water and the sections were counterstained with Mayer's hematoxylin, dehydrated with gradient alcohol, mounted with neutral balsam. The immunoreactivity of each slice was examined by three independent investigators and photographed under the light microscope (Leica DM2000, Germany) with digital camera at high magnification (Leica DFC450C, Germany). The staining immunoreactivity was scored ranged from $0 \%$ to $100 \%$ ( $5 \%$ as intervals) by calculating the proportion of positive cancerous cells over all areas and the scorers were blinded for each other' score. A cutoff point of each marker was chosen by using median value to dichotomize cases. Scoring above or below the cutoff point was classified as "elevated" or "decreased", respectively.

Table 1. Clinicopathological variables and the expressions of c-Myb and COX-2 in patients with colorectal cancer

\begin{tabular}{|c|c|c|c|c|c|c|c|}
\hline \multirow[t]{2}{*}{ Characteristics } & \multirow{2}{*}{$\begin{array}{l}\text { Total } \\
\text { (n) }\end{array}$} & \multicolumn{3}{|c|}{ Tumor c-Myb expression } & \multicolumn{3}{|c|}{ Tumor COX-2 expression } \\
\hline & & Elevated (n) & Decreased (n) & $\mathrm{P}$-value & Elevated (n) & Decreased (n) & P-value \\
\hline All cases & 202 & 116 & 86 & & 102 & 100 & \\
\hline Age (years) & & & & 0.933 & & & 0.946 \\
\hline$<60$ & 57 & 33 & 24 & & 29 & 28 & \\
\hline$\geq 60$ & 145 & 83 & 62 & & 73 & 72 & \\
\hline Gender & & & & 0.894 & & & 0.195 \\
\hline Female & 88 & 51 & 37 & & 49 & 39 & \\
\hline Male & 114 & 65 & 49 & & 53 & 61 & \\
\hline Tumor location & & & & 0.095 & & & 0.030 \\
\hline Right hemicolon & 76 & 51 & 25 & & 47 & 29 & \\
\hline Left hemicolon & 61 & 32 & 29 & & 29 & 32 & \\
\hline Rectum & 65 & 33 & 32 & & 26 & 39 & \\
\hline Differentiation & & & & 0.067 & & & 0.150 \\
\hline Well-moderate & 184 & 102 & 82 & & 90 & 94 & \\
\hline Poor & 18 & 14 & 4 & & 12 & 6 & \\
\hline Tumor size $(\mathrm{cm})$ & & & & 0.812 & & & 0.199 \\
\hline$<5$ & 110 & 64 & 46 & & 51 & 59 & \\
\hline$\geq 5$ & 92 & 52 & 40 & & 51 & 41 & \\
\hline Depth of invasion & & & & 0.020 & & & 0.003 \\
\hline Tis - T2 & 23 & 8 & 15 & & 5 & 18 & \\
\hline $\mathrm{T} 3-\mathrm{T} 4$ & 179 & 108 & 71 & & 97 & 82 & \\
\hline Lymph node involvement & & & & 0.062 & & & 0.292 \\
\hline N0 & 126 & 66 & 60 & & 60 & 66 & \\
\hline $\mathrm{N} 1-\mathrm{N} 2$ & 76 & 50 & 26 & & 42 & 34 & \\
\hline Distant metastatic spread & & & & 0.092 & & & 0.370 \\
\hline Absence (M0) & 191 & 107 & 84 & & 95 & 96 & \\
\hline Presence (M1) & 11 & 9 & 2 & & 7 & 4 & \\
\hline TNM stage & & & & & & & \\
\hline $0-\Pi$ & 122 & 62 & 60 & 0.019 & 56 & 66 & 0.107 \\
\hline III - IV & 80 & 54 & 26 & & 46 & 34 & \\
\hline 5-Fu-based chemotherapy & & & & 0.654 & & & 0.481 \\
\hline Absence & 100 & 59 & 41 & & 53 & 47 & \\
\hline Presence & 102 & 57 & 45 & & 49 & 53 & \\
\hline KI-67 expression & & & & 0.409 & & & 0.153 \\
\hline$<50 \%$ & 71 & 38 & 33 & & 31 & 40 & \\
\hline$\geq 50 \%$ & 131 & 78 & 53 & & 71 & 60 & \\
\hline P53 status & & & & 0.808 & 28 & 36 & 0.192 \\
\hline Negative & 64 & 36 & 28 & & 74 & 64 & \\
\hline Positive & 138 & 80 & 58 & & & & \\
\hline CEA status & & & & 0.569 & & & 0.778 \\
\hline Negative & 101 & 56 & 45 & & 50 & 51 & \\
\hline Positive & 101 & 60 & 41 & & 52 & 49 & \\
\hline
\end{tabular}

Abbreviations: TNM, tumor node metastasis 


\section{Western blotting analysis}

Total protein were obtained from three pairs of matched normal and cancerous CRC frozen tissues using RIPA Lysis Buffer (Beyotime, Shanghai, China) according to the specification. Total protein was quantified using a BCA kit (TIANGEN, Beijing, China). SDS-PAGE was performed for $0.5 \mathrm{~h}$ at $80 \mathrm{~V}$ and then at $120 \mathrm{~V}$ for $1.5 \mathrm{~h}$. After electrophoresis, the separated proteins were transferred from the gel to the PVDF membranes (Millipore, Bedford, USA) at $250 \mathrm{~mA}$ for $2 \mathrm{~h}$ at $4^{\circ} \mathrm{C}$. Then the PVDF was blocked with skim milk in tris-buffered saline tween (TBST) 1 $\mathrm{h}$ at room temperature. Following blocking, the membranes was incubated overnight at $4^{\circ} \mathrm{C}$ with the primary antibody: anti-c-Myb (Rabbit polyclonal, 1:500, Abcam, Cambridge, United Kingdom, ab117 635); anti-COX-2 (Rabbit monoclonal, 1:500, Abcam, Cambridge, United Kingdom, ab62331); anti-beta Actin (Mouse monoclonal, 1:5000, SIGMA, California, USA, A5441). After washing with TBST three times (5 min per wash), the PVDF was incubated with the corresponding secondary antibody (anti-rabbit, 1:5000, LICOR, Nebraska, USA, 926-32223; antimouse, 1:5000, LICOR, Nebraska, USA, 926-68072) $1 \mathrm{~h}$ at room temperature and analyzed with the Odyssey 3.0 system.

\section{Statistical analysis}

All statistical analyses were performed by using the IBM SPSS Statistics 20.0 software (IBM, lnc., Chicago, Illinois, USA) and GraphPad Prism 6 software (GraphPad software, lnc., San Diego, California, USA). For comparison of expression of each marker between the CRC tissues and the matched adjacent normal tissues, a paired Student's $t$ test was conducted. The proportion values of positively stained cells were transformed using the arcsine-square-root transformation to normalize the data[21]. The relationships between c-Myb and COX-2 expression was analyzed using linear or non-linear regression, if appropriate. The associations between the clinicopathological data and the staining of c-Myb and COX-2 were performed using Pearson's Chi-square. The impacts of clinical variables and the staining of c-Myb and COX-2 were estimated by using univariate and multivariate Cox proportional hazards regression model. All tests were double tail and the $p$ values below 0.05 were considered significant.

\section{Results}

\section{Detection of c-Myb and COX-2 expression in CRC tissues and paired adjacent mucosa}

Two hundred and two cases with matched normal and neoplastic tissues from CRC patients were included in our present study. Representative IHC staining of $\mathrm{c}-\mathrm{Myb}$ and COX-2 for CRC tissues and paired adjacent mucosa are represented in Figure $\mathbf{1}$. Immunoreactivity for c-Myb was detected primarily in the nuclei, while COX-2 was expressed primarily in the cytoplasm. The median staining score of c-Myb was $85 \%$ in the CRC tissues and $30 \%$ in the matched adjacent normal tissues. Meanwhile, the median staining score of COX-2 was $60 \%$ in the cancerous tissues and $20 \%$ in the matched noncancerous tissues. In agreement with the reported studies[9,22,23], the expression levels of both c-Myb $(\mathrm{p}<0.001)$ and COX-2 $(p<0.001)$ were higher in the CRC tissues in comparison with the adjacent normal tissues (Figure 2A \& B).

Furthermore, linear regression model was examined to explore the association between c-Myb and COX-2 expression. A statistically significant correlation was found between the proportional immunostaining level of c-Myb and the expression of COX-2 ( $r=0.391, p<0.001$; Figure 2C). We further validated the result by Western blot. Compared with noncancerous tissues, the protein expression of c-Myb was found to be upregulated in the CRC tissues, accompanied by high expression of COX-2 (Figure 2D).

\section{The associations of c-Myb and COX-2 expression with clinicopathological variables}

To explore the relationship of c-Myb and COX-2 expression with clinicopathological variables, the median value of each marker was chosen as the cutoff point to dichotomize the staining scores. With this criterion, 116 cases were classified as high-expression and 86 cases as low-expression for c-Myb. Likewise, 102 cases were classified as high-expression and 100 cases as low-expression for COX-2.

As demonstrated in Table 1, elevated c-Myb expression was significantly associated with more advanced tumor invasion $(p=0.020)$ and higher TNM stage $(p=0.019)$. Although the elevated $\mathrm{c}-\mathrm{Myb}$ expression was slightly associated with tumor differentiation $(\mathrm{p}=0.067)$ and lymph node involvement $(p=0.062)$, the results didn't reach statistical significance. Elevated COX-2 expression was also significantly correlated with more advanced tumor invasion $(\mathrm{p}=0.003)$ and tumor location $(\mathrm{p}=$ 0.030). Among variables including age, gender, tumor differentiation, tumor size, lymph node involvement, distant metastatic spread, TNM stage, 5-Fu-based chemotherapy, KI-67 expression, P53 status and CEA status, there was no association between these variables and the COX-2 expression. 


\section{Expression of c-Myb and COX-2 correlated with poor overall survival in colorectal carcinoma}

Univariate associations of the expression of each protein with overall survival were listed in Table 2. Poorer tumor differentiation (Hazard ratio (HR), 2.348; 95\% confidence interval (95\% CI), 1.272 - 4.333; $\mathrm{p}=0.006)$, more depth of invasion (HR, $4.324 ; 95 \% \mathrm{CI}$, 1.365 - 13.698; $\mathrm{p}=0.013)$, positive lymph node involvement (HR, 4.000; 95\% CI, 2.545 - 6.286; $\mathrm{p}<$ 0.001), positive distant metastatic spread (HR, 3.407; 95\% CI, 1.752 - 6.623; $\mathrm{p}<0.001)$, higher TNM stage (HR, 2.637; 95\% CI, 1.975 - 3.520; $\mathrm{p}<0.001$ ), and positive CEA status (HR, 1.583; 95\% CI, 1.019 - 2.458; $\mathrm{p}=0.041$ ) demonstrated shorter overall survival of CRC. Cases with c-Myb overexpression, as determined by IHC, had a 2.693 -fold (95\% CI, 1.640 4.421; $\mathrm{p}<0.001$ ) more risk for death (Figure 3A). Meanwhile, cases with COX-2 overexpression had a 2.334-fold (95\% CI, 1.485 - 3.669; p < 0.001) poorer overall survival (Figure 3D).

Multivariate Cox proportional hazards regression analysis was performed using all 202 cases with complete clinical data for variables that were significant on univariate model (Table 2). After multivariable adjustment by considering the clinicopathological variables including tumor differentiation, depth of invasion, lymph node involvement, distant metastatic spread, TNM stage, and CEA status, only the lymph node involvement (HR, 3.535; 95\% CI, $1.127-11.084 ; \mathrm{p}=0.030)$, distant metastatic spread (HR, 2.247; 95\% CI, $1.014-4.978 ; \mathrm{p}=0.046$ ), and expression of $\mathrm{c}-\mathrm{Myb}$ and $\mathrm{COX}-2$ remained independently significant. Tumor tissues with elevated c-Myb expression had a 1.841 -fold $(95 \% \mathrm{CI}$, $1.082-3.133 ; p=0.024)$ more risk for death. Tumor tissues with COX-2 overexpression had a 1.672-fold (95\% CI, 1.022 - 2.736; $\mathrm{p}<0.041)$ poorer overall survival.

To further investigate the influence of c-Myb and COX-2 expression on patients with adjuvant chemotherapy, we conducted subgroup analysis in patients with or without 5-Fu-based chemotherapy. Elevated expression of c-Myb or COX-2 had a worse response to 5-Fu-based chemotherapy $(\mathrm{p}<0.001$ and $p=0.001$, respectively; Figure 3C, 3F) in comparison with patients without chemotherapy $(\mathrm{p}=0.024$ and $\mathrm{p}$ $=0.039$, respectively; Figure 3B, 3E).

\section{Combination of c-Myb and COX-2 in predicting prognosis of CRC}

To confirm that the combination of c-Myb and COX-2 may help predict prognosis of CRC patients, we constructed three subgroups based on the expression levels of both genes: subgroup 1, low c-Myb and low COX-2; subgroup 2, (low c-Myb and high COX-2) or (high c-Myb and low COX-2); subgroup 3, high c-Myb and high COX-2. As expected, overexpression of both genes predicted worse overall survival ( $p<0.001$; Figure $4 \mathrm{~A})$. When stratified by 5 -Fu-based chemotherapy, the promising prognostic model by combination of $\mathrm{c}-\mathrm{Myb}$ and COX-2 were yet again confirmed, suggesting that the two markers may help to predict and select the effect of chemotherapy on survival time in patients with CRC (Figure 4B, 4C).
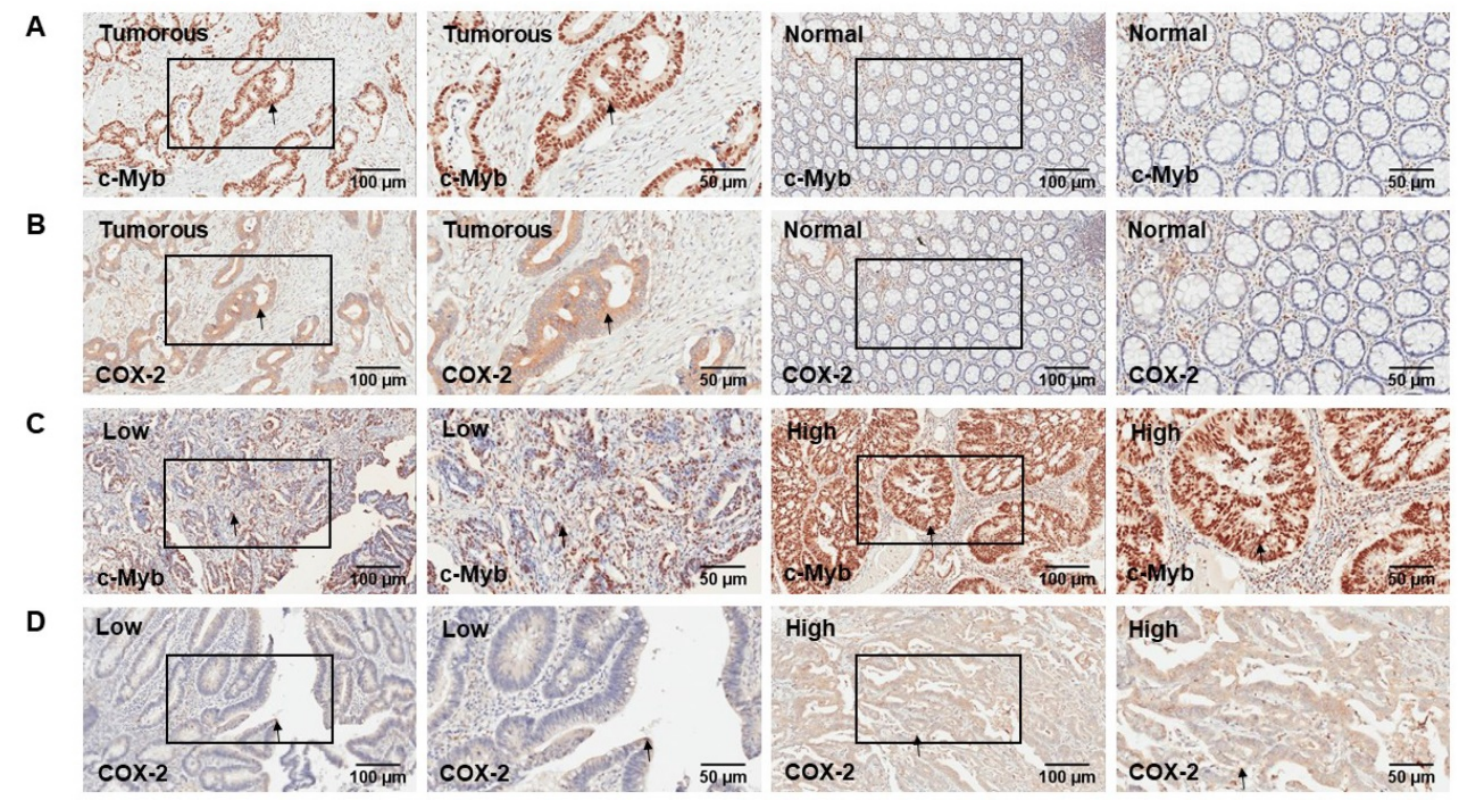

Figure 1. Representative IHC staining of c-Myb and COX-2 for CRC tissues and paired adjacent mucosa. A) IHC of c-Myb protein in tumorous or adjacent normal tissues. B) IHC of COX-2 protein in tumorous or adjacent normal tissues. C) Low and high expression level of c-Myb protein in tumorous tissues. D) Low and high expression level of COX-2 protein in tumorous tissues. Original magnifications: $\times 100$ or $\times 200$. 


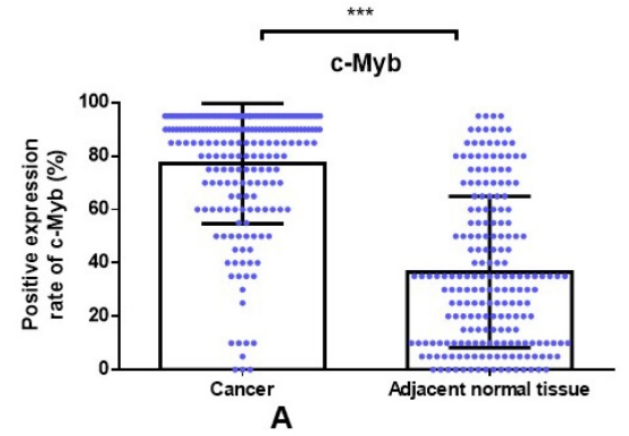

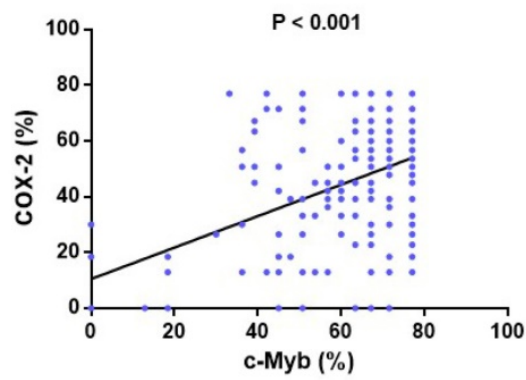

C

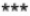

\section{cox-2}
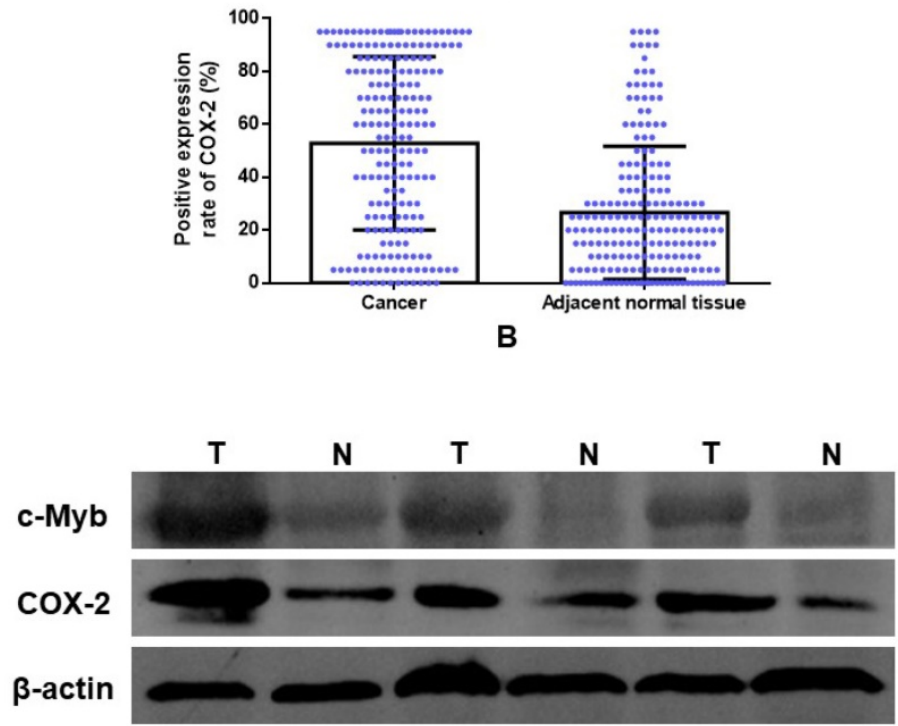

D

Figure 2. The positive expression rate of c-Myb and COX-2 in CRC. A) IHC detection of c-Myb in 202 paired of CRC and noncancerous tissues (paired Student's t test; ***, p < 0.001); B) IHC detection of COX-2 in 202 paired of CRC and noncancerous tissues (paired Student's t test; ***, p < 0.001 ). C) Correlation of c-Myb expression with COX-2 expression (linear regression; $r=0.391, p<0.001$ ). D) Western blot analysis of the protein expression of $c-M y b$ and COX-2 in the CRC tissues, as compared with noncancerous tissues.

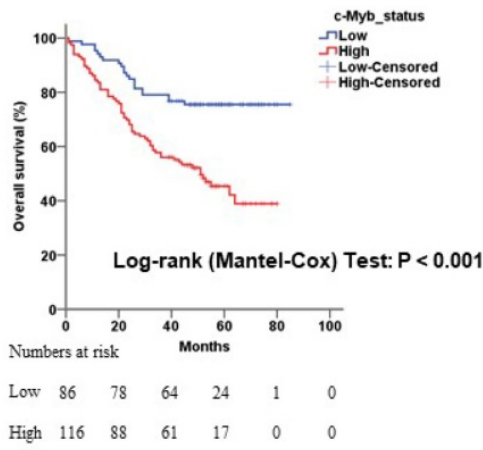

A

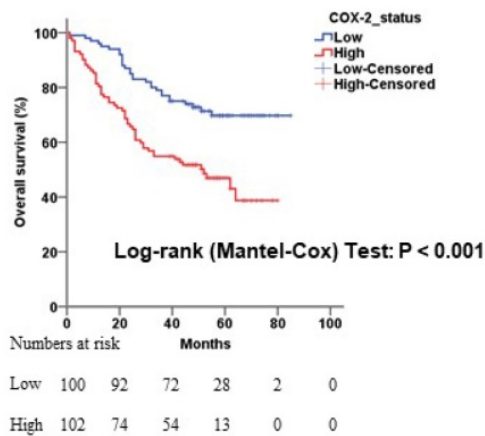

D

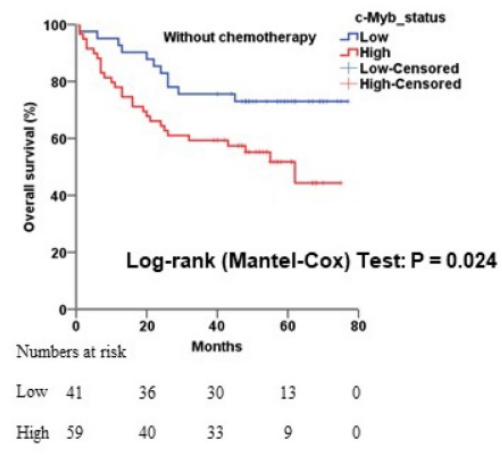

B

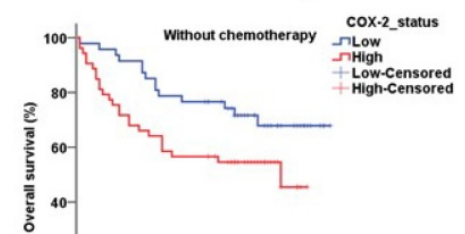

Log-rank (Mantel-Cox) Test: $P=0.039$

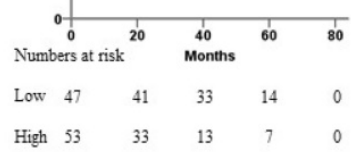

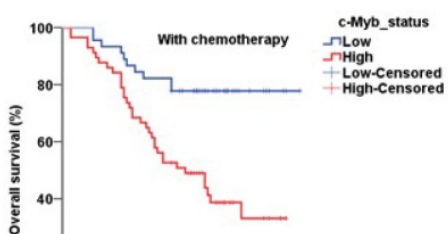

Log-rank (Mantel-Cox) Test: $P<0.001$

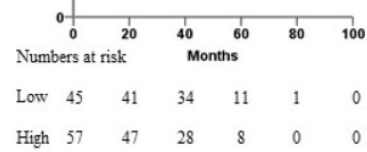

C

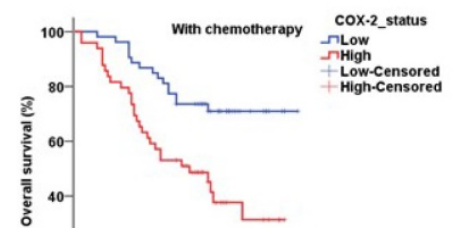

Log-rank (Mantel-Cox) Test: $P=0.001$

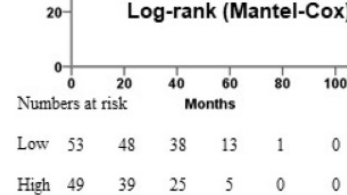

$\mathbf{F}$

Figure 3. The influence of c-Myb and COX-2 expression for overall survival in CRC. A) Relationship of c-Myb expression to overall survival in all 202 patients (log-rank; $\mathrm{p}<0.001$ ). B) Relationship of c-Myb expression to overall survival in patients without 5-Fu-based chemotherapy (log-rank; $p=0.024)$. C) Relationship of c-Myb expression to overall survival in patients with 5 -Fu-based chemotherapy (log-rank; $P<0.001$ ). D) Relationship of COX-2 expression to overall survival in all 202 patients $(\log$-rank; $\mathrm{P}<0.001)$. E) Relationship of COX-2 expression to overall survival in patients without 5-Fu-based chemotherapy (log-rank; $P=0.039)$. F) Relationship of COX-2 expression to overall survival in patients with 5-Fu-based chemotherapy (log-rank; $p=0.001$ ) 

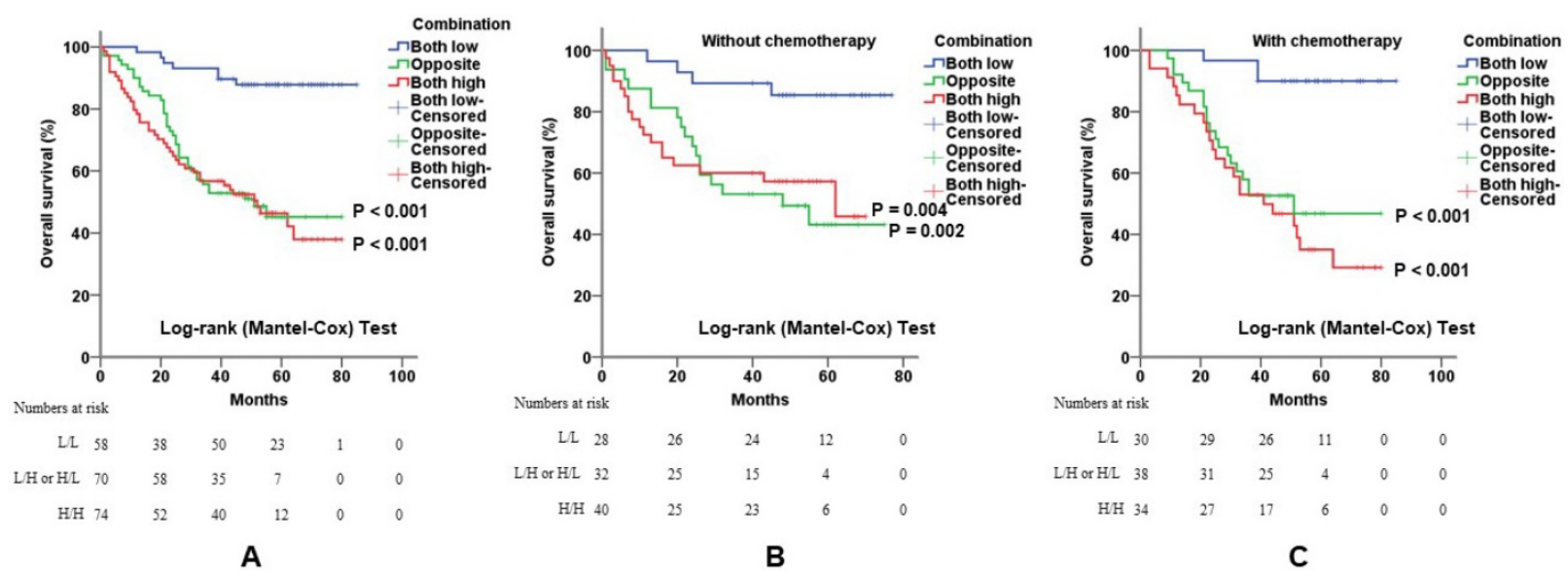

Figure 4. Combination of c-Myb and COX-2 in predicting prognosis of CRC. A, B and C) Combination of both up-regulated molecules predicted worse overall survival in all patients ( $A$; log-rank; $p<0.001$ for subgroup 2 and $p<0.001$ for subgroup 3 , respectively) or in patients without 5 -Fu-based chemotherapy $(B ;$ log-rank; $p=0.002$ for subgroup 2 and $p=0.004$ for subgroup 3, respectively) or in patients with 5-Fu-based chemotherapy (C; log-rank; $p<0.001$ for subgroup 2 and $p<0.001$ for subgroup 3 , respectively). Subgroup 1, low c-Myb and low COX-2; subgroup 2, (low c-Myb and high COX-2) or (high c-Myb and low COX-2); subgroup 3, high c-Myb and high COX-2. Subgroup 1 was chosen as control.

Table 2 The effects of clinicopathologic variables on overall survival rate by univariate and multivariate Cox proportional hazard regression analysis

\begin{tabular}{|c|c|c|c|c|c|c|}
\hline \multirow[t]{2}{*}{ Variables } & \multicolumn{3}{|c|}{ Univariate } & \multicolumn{3}{|c|}{ Multivariate } \\
\hline & HR & $95 \% \mathrm{CI}$ & P-value & HR & $95 \% \mathrm{CI}$ & P-value \\
\hline c-Myb & 2.693 & $1.640-4.421$ & $<0.001$ & 1.841 & $1.082-3.133$ & 0.024 \\
\hline COX-2 & 2.334 & $1.485-3.669$ & $<0.001$ & 1.672 & $1.022-2.736$ & 0.041 \\
\hline Age & 1.033 & $0.643-1.661$ & 0.892 & & & \\
\hline Gender & 0.957 & $0.621-1.476$ & 0.843 & & & \\
\hline Tumor location & 0.910 & $0.698-1.185$ & 0.482 & & & \\
\hline Differentiation & 2.348 & $1.272-4.333$ & 0.006 & 1.409 & $0.749-2.651$ & 0.288 \\
\hline Tumor size & 1.192 & $0.775-1.833$ & 0.425 & & & \\
\hline Depth of invasion & 4.324 & $1.365-13.698$ & 0.013 & 1.995 & $0.612-6.500$ & 0.252 \\
\hline Lymph node involvement & 4.000 & $2.545-6.286$ & $<0.001$ & 3.535 & $1.127-11.084$ & 0.030 \\
\hline Distant metastatic spread & 3.407 & $1.752-6.623$ & $<0.001$ & 2.247 & $1.014-4.978$ & 0.046 \\
\hline TNM stage & 2.637 & $1.975-3.520$ & $<0.001$ & 0.945 & $0.285-3.134$ & 0.927 \\
\hline 5-Fu-based chemotherapy & 1.038 & $0.674-1.598$ & 0.865 & & & \\
\hline KI-67 expression & 1.212 & $0.762-1.928$ & 0.417 & & & \\
\hline TP53 status & 1.318 & $0.814-2.135$ & 0.262 & & & \\
\hline CEA status & 1.583 & $1.019-2.458$ & 0.041 & 1.258 & $0.795-1.990$ & 0.326 \\
\hline
\end{tabular}

Abbreviations: TNM, tumor node metastasis; HR, hazard ratio; $\mathrm{CI}$, confidence interval

5-Fu-based adjuvant chemotherapy is established routine therapy for CRC patients with stage II and stage III disease, especially for those with poor prognostic features. When further stratified by TNM stage, the co-overexpression of both genes was still of value for prognostic evaluation. In detail, overexpression of c-Myb and COX-2 was significantly associated with shorter overall survival for stage II $(p=0.028$; Figure 5A, 5B) and stage III ( $p=0.003$; Figure 5C, 5D) patients with $5-\mathrm{Fu}$ based chemotherapy.

\section{Discussion}

Although our previous study identified the oncogenic role of c-Myb in cellular apoptosis, progression and proliferation in cultured cells and an animal model, its clinical significance remains unclear and requires interrogation[7]. In this study, we have firstly developed a prognostic model based on two proteins to enhance and better predict the prognosis of CRC. Our study presented that the proportional immunostaining level of $\mathrm{c}-\mathrm{Myb}$ was significantly correlated with the expression of COX-2. The expression level of c-Myb in combination with COX-2 can fairly well categorize CRC cases into low- and high-risk phenotypes with difference in overall survival rate. Cases with high c-Myb and COX-2 were involved in worse response to chemotherapy.

The MYB family of the transcription factor genes contain c-Myb, b-Myb and a-Myb[24]. All members of the MYB family contain a conserved DNA-binding domain[24]. Compelling studies have shown that c-Myb plays a pivotal role of proto-oncogenes in cancers including CRC[25]. However, a recent study published by Michal Tichý found a negative association between the expression of c-Myb in CRC tissue and the presence of distant metastases[26]. Considering that both pro-invasive and antimetastatic roles of c-Myb were described, we examined the expression of $\mathrm{c}-\mathrm{Myb}$ at protein level in 202 CRC tissue samples and verified it at mRNA level using public databases from The Cancer Genome Atlas (TCGA) cohort of patients with colon 
adenocarcinoma (TCGA COAD). In agreement with the reported studies[9,22,27], we found that the expression of c-Myb ( $\mathrm{p}<0.001)$, detected by IHC, was significantly higher in the CRC tissues in comparison with the adjacent normal tissues. Meanwhile, elevated expression of c-Myb was significantly associated with more advanced tumor invasion $(p=0.020)$, higher TNM stage $(p=0.019)$ and poorer overall survival ( $p$ $<$ 0.001). Whereas, there were no significant association between the amount of the $c-M y b$ mRNA and the poor outcome of patients with CRC according to the TCGA COAD (Supplementary table S1). These findings supported the fact that the protein level of the $c-M y b$, but not the amount of the gene mRNA, may serve as a good prognostic marker.

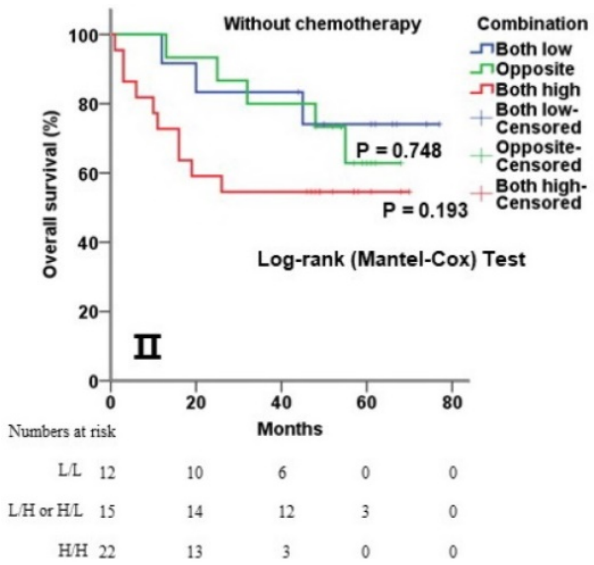

A

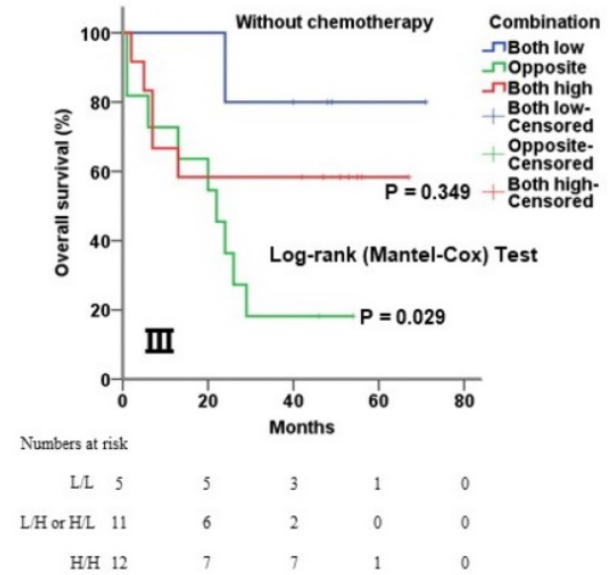

As a nuclear transcription factor, c-Myb participates in diverse cellular processes by up-regulation of its downstream transcriptional targets including COX-2[28-30]. COX-2 inhibits apoptosis and promotes cell proliferation in numerous cancers including breast cancer[31,32], cervical cancer[33], lung cancer[34], gastric cancer[35], and CRC[36]. A myriad of literature has confirmed that long-term therapy with non-steroidal anti-inflammatory drugs (NSAIDs) such as aspirin, or selective COX-2 inhibitors like celecoxib, are associated with a reduced risk of $\mathrm{CRC}[36-39]$. When it comes to the prognostic value, the COX2 expression by IHC was found to have a significant correlation with tumor stage, despite few studies demonstrated a negative effect[40-42].

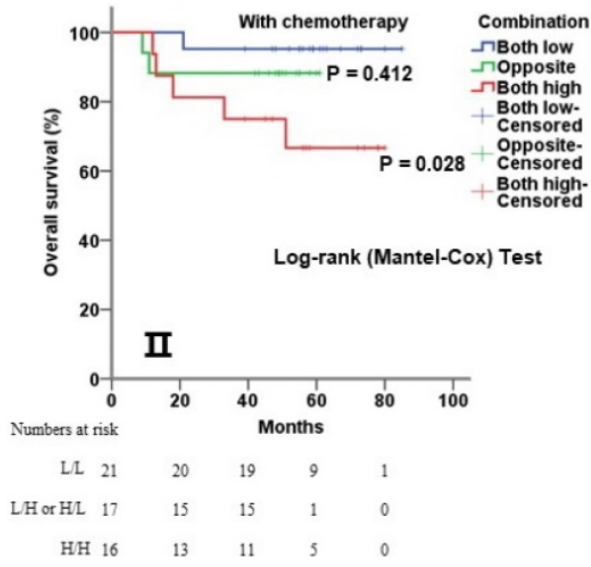

B

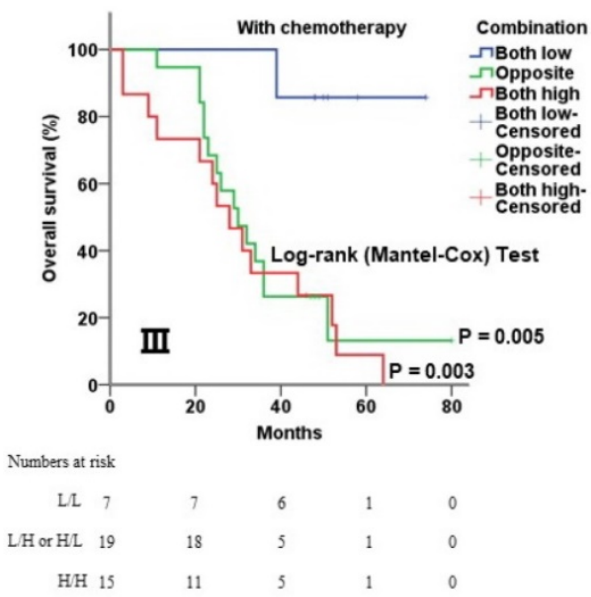

C

Figure 5. Combination of c-Myb and COX-2 in predicting prognosis of CRC in stage II and III patients. A and B) Combination of both up-regulated molecules predicted worse overall survival in stage II patients without (A; log-rank; $p=0.748$ for subgroup 2 and $p=0.193$ for subgroup 3 , respectively) or with 5 -Fu based chemotherapy ( $B$; log-rank; $p=0.412$ for subgroup 2 and $p=0.028$ for subgroup 3 , respectively). $C$ and $D$ ) Combination of both up-regulated molecules predicted worse overall survival in stage III patients without ( $C$; log-rank; $p=0.029$ for subgroup 2 and $p=0.349$ for subgroup 3, respectively) or with 5-Fu based chemotherapy ( $D$; log-rank; $p<0.001$ for subgroup 2 and $p<0.001$ for subgroup 3, respectively). Subgroup 1, low c-Myb and low COX-2; subgroup 2, (low c-Myb and high COX-2) or (high c-Myb and low COX-2); subgroup 3, high c-Myb and high COX-2. Subgroup 1 was chosen as control. 
Ramsay and his colleagues were the first to demonstrate that human COX-2 promoter contains a high-affinity binding site of c-Myb. Given that both c-Myb and COX-2 drive tumor progression[29], we have reason to believe that using c-Myb-COX-2 partner model to predict CRC prognosis may be better than utilizing any of the single indicator. A recent study had found that the combination of c-Myb, v-kit Hardy-Zuckerman 4 feline sarcoma viral oncogene homolog (c-kit), and COX-2 show better clinical significance in predicting prognosis in salivary adenoid cystic carcinoma[43]. However, the relationship between c-Myb and COX-2 in CRC hasn't been verified in clinic specimens up to now. In this context our findings have important clinical significance and urge us to confirm the prognostic value of c-Myb-COX-2 partner model in CRC. In our study, both univariate and multivariate Cox proportional hazards regression analysis supported that cases with high c-Myb and COX-2 indicated poorer overall survival. When stratified by 5 -Fu-based chemotherapy status and TNM stage, the prognostic value by combination of $\mathrm{c}-\mathrm{Myb}$ and COX-2 were reconfirmed and validated. These findings suggest that the overexpression of both c-Myb and COX-2 is of prognostic screening value in patients with CRC. However, according to the TCGA COAD database, there were no significant association between the mRNA level of both gene and the prognosis of CRC (Supplementary table S2). The reason for this could be that c-Myb and COX2 promotes the progression of the disease mainly at the posttranscriptional level. Studies based on gain-of-functions/loss-of-functions experiments are required to further explain this phenomenon.

In conclusion, our present study demonstrates that both the c-Myb and COX-2 are two independent prognostic biomarkers and the c-Myb-COX-2 model reinforces the function in prognostic assessment in patients with CRC.

\section{Abbreviations}

CEA, carcinoembryonic antigen; c-Myb, v-myb avian myeloblastosis viral oncogene homolog; COX-2, prostaglandin-endoperoxide synthase 2; CRC, colorectal cancer; HR, hazard ratio; 95\% CI, 95\% confidence interval; IHC, immunohistochemistry; NSAIDs, nonsteroidal anti-inflammatory drugs; PBS, phosphate buffer saline; TBST, tris-buffered saline tween TMA, tissue microarray; TNM, tumor node metastasis; WB, western blot.

\section{Supplementary Material}

Supplementary tables.

http://www.jcancer.org/v10p1601s1.pdf

\section{Acknowledgments}

We would like to express our deep and sincere gratitude to the patients and clinicians for their contributions to this study.

\section{Funding}

This work was supported by grants from the Fudan Outstanding Young Talent Training Plan (YJYQ201601), Sponsored by the National Natural Science Foundation of China (No. 81802412), Shanghai Sailing Program (No.18YF1414900), Shanghai Pujiang Program (17PJD007), and Shanghai Youth Top-notch Talent Support Program (Yanlei Ma).

\section{Authors' contributions}

Y.Y., Y.M. and X.L. designed the study. Y.Y., R.X., H.Z., H.L., J.G. and Q.W. performed the experiments. H.Z., Q.W. and R.T. collected the clinical samples. Y.M. Q.W. and X.L. supervised specific experiments and revised the manuscript. Y.Y. and R.X. wrote the manuscript. All authors read and approved the final manuscript.

\section{Ethics approval and consent to participate}

All procedures performed in studies involving human participants were in accordance with the ethical standards of the Research Ethics Committee of the related institute.

\section{Competing Interests}

The authors have declared that no competing interest exists.

\section{References}

1. Song M, Nishihara R, Wang M, Chan AT, Qian ZR, Inamura $K$, Zhang X, Ng K, Kim SA, Mima K. Plasma 25-hydroxyvitamin D and colorectal cancer risk according to tumour immunity status. Gut. 2015; 65:296-304.

2. Chen W, Zheng R, Baade PD, Zhang S, Zeng H, Bray F, Jemal A, Yu XQ, He J. Cancer statistics in China, 2015. Ca A Cancer Journal for Clinicians. 2016; 66:115.

3. Lin J, Qiu M, Xu R, Dobs AS. Comparison of survival and clinicopathologic features in colorectal cancer among African American, Caucasian, and Chinese patients treated in the United States: Results from the surveillance epidemiology and end results (SEER) database. Oncotarget. 2015; 6:33935-33943.

4. Fakih MG. Metastatic colorectal cancer: Current state and future directions. Journal of Clinical Oncology Official Journal of the American Society of Clinical Oncology. 2015; 33:1809-1824.

5. Kneuertz PJ, Chang GJ, Hu CY, Rodriguezbigas MA, Eng C, Vilar E, Skibber JM, Feig BW, Cormier JN, You YN. Overtreatment of young adults with colon cancer: More intense treatments with unmatched survival gains. JAMA Surg. $2015 ; 150: 402$.

6. Guinney J, Dienstmann R, Wang X, Reyniès AD, Schlicker A, Soneson C, Marisa L, Roepman P, Nyamundanda G, Angelino P. The consensus molecular subtypes of colorectal cancer. Nat Med. 2015; 21:1350.

7. Feng J, Yang Y, Zhang P, Wang F, Ma Y, Qin H, Wang Y. MiR-150 functions as a tumour suppressor in human colorectal cancer by targeting c-Myb. Journal of Cellular \& Molecular Medicine. 2014; 18:2125.

8. Ma Y, Zhang P, Wang F, Zhang H, Yang J, Peng J, Liu W, Qin H. MiR-150 as a potential biomarker associated with prognosis and therapeutic outcome in colorectal cancer. Gut. 2012; 61:1447.

9. Ramsay RG, Gonda TJ. MYB function in normal and cancer cells. Nat Rev Cancer. 2008; 8:523.

10. Slamon DJ, Boone TC, Murdock DC, Keith DE, Larson RA, Souza LM. Studies of the human c-myb gene and its product in human acute leukemias. Science. 1986; 233:347. 
11. Mitani Y, Li J, Rao PH, Zhao YJ, Bell D, Lippman SM, Weber RS, Caulin C, Elnaggar AK. Comprehensive analysis of the MYB-NFIB gene fusion in salivary adenoid cystic carcinoma: Incidence, variability, and clinicopathologic significance. Clin Cancer Res. 2010; 16:4722.

12. Furth PA, Nakles RE, Millman S, Diaz-Cruz ES, Cabrera MC. Signal transducer and activator of transcription 5 as a key signaling pathway in normal mammary gland developmental biology and breast cancer. Breast Cancer Res. 2011; 13:220.

13. Pereira LA, Hugo HJ, Malaterre J, Huiling X, Sonza S, Cures A, Purcell DF, Ramsland PA, Gerondakis S, Gonda TJ. MYB elongation is regulated by the nucleic acid binding of NFKB p50 to the intronic stem-loop region. Plos One. 2014; 10:e122919.

14. Nd BL, Kanner WA, Fehr A, Andrén Y, Moskaluk CA, Löning T, Stenman G, Jr FH. Analysis of MYB expression and MYB-NFIB gene fusions in adenoid cystic carcinoma and other salivary neoplasms. Modern Pathology An Official Journal of the United States \& Canadian Academy of Pathology Inc. 2011; 24:1169-1176.

15. Thompson MA, Rosenthal MA, Ellis SL, Friend AJ, Zorbas MI, Whitehead RH, Ramsay RG. C-Myb down-regulation is associated with human colon cell differentiation, apoptosis, and decreased Bcl-2 expression. Cancer Res. 1998; 58:5168-5175.

16. Bian Z, Li L, Cui J, Zhang H, Liu Y, Zhang CY, Ke Z. Role of miR-150-targeting $\mathrm{c}-\mathrm{Myb}$ in colonic epithelial disruption during dextran sulphate sodium-induced murine experimental colitis and human ulcerative colitis. J Pathol. 2011; 225:544.

17. Ernst M, Ramsay RG. Colorectal cancer mouse models: Integrating inflammation and the stroma. Journal of Gastroenterology \& Hepatology. $2012 ; 27 \cdot 39-50$

18. X J, X Í, Vec, Ergang P, Mandys V, Kment M, X J, Pácha Í. Expression profiles of proliferative and antiapoptotic genes in sporadic and colitis-related mouse colon cancer models. Int J Exp Pathol. 2010; 91:44-53.

19. Ng K, Meyerhardt JA, Chan AT, Sato K, Chan JA, Niedzwiecki D, Saltz LB, Mayer RJ, Rd BA, Schaefer PL. Aspirin and COX-2 inhibitor use in patients with stage III colon cancer. Journal of the National Cancer Institute. 2014; 107:345

20. Brown JR, DuBois RN. COX-2: A molecular target for colorectal cancer prevention. J Clin Oncol. 2005; 23:2840-2855.

21. Rasiah KK, Kench JG, Gardinergarden M, Biankin AV, Golovsky D, Brenner PC, Kooner R, O'Neill GF, Turner JJ, Delprado W. Aberrant neuropeptide Y and macrophage inhibitory cytokine-1 expression are early events in prostate cancer development and are associated with poor prognosis. Cancer epidemiology, biomarkers \& prevention : a publication of the American Association for Cancer Research, cosponsored by the American Society of Preventive Oncology. 2006; 15:711.

22. Biroccio A, Benassi B, D'Agnano I, D'Angelo C, Buglioni S, Mottolese M, Ricciotti A, Citro G, Cosimelli M, Ramsay RG. C-Myb and bcl-x overexpression predicts poor prognosis in colorectal cancer. Am J Pathol. 2001; 158:1289-1299.

23. Roelofs HM, Morsche RHT, Heumen BWV, Nagengast FM, Peters WH. Over-expression of COX-2 mRNA in colorectal cancer. BMC Gastroenterol. 2014; 14:1-6.

24. Tichý M, Knopfová L, Jarkovský J, Pekarčíková L, Veverková L, Vlček P, Katolická J, Čapov I, Hermanová M, Šmarda J. Overexpression of c-Myb is associated with suppression of distant metastases in colorectal carcinoma. Tumor Biol. 2016; 50:1-7.

25. Elzagheid A, Emaetig F, Alkikhia L, Buhmeida A, Syrjänen K, Elfaitori O, Latto M, Collan Y, Pyrhönen S. High cyclooxygenase-2 expression is associated with advanced stages in colorectal cancer. Anticancer Res. 2013; 33:3137-3143.

26. Eldeiry WS, Vijayvergia N, Xiu J, Scicchitano A, Lim B, Yee NS, Harvey HA, Gatalica Z, Reddy S. Molecular profiling of 6,892 colorectal cancer samples suggests different possible treatment options specific to metastatic sites. Cancer Biol Ther. 2015; 16:1726.

27. Du P, Xu B, Zhang D, Shao Y, Zheng X, Li X, Xiong Y, Wu C, Jiang J. Hierarchical investigating the predictive value of p53, COX2, EGFR, nm23 in the post-operative patients with colorectal carcinoma. Oncotarget. 2016;

28. Oh IH, Reddy EP. The myb gene family in cell growth, differentiation and apoptosis. Oncogene. 1999; 18:3017.

29. Malaterre J, Pereira L, Putoczki T, Millen R, Paquet-Fifield S, Germann M, Liu J, Cheasley D, Sampurno S, Stacker SA. Intestinal-specific activatable Myb initiates colon tumorigenesis in mice. Oncogene. 2015; 35:2475-2484.

30. Greco C, Alvino SS, Assisi D, Lapenta R, Grassi A, Stigliano V, Mottolese M, Casale V. Activation of c-MYC and c-MYB proto-oncogenes is associated with decreased apoptosis in tumor colon progression. Anticancer Res. 2001; 21:3185-3192.

31. Ramsay RG, Friend A, Vizantios Y, Freeman R, Sicurella C, Hammett F, Armes J, Venter D. Cyclooxygenase-2, a colorectal cancer nonsteroidal anti-inflammatory drug target, is regulated by c-MYB. Cancer Res. 2000; 60:1805-1809.

32. Hugo HJ, Saunders C, Ramsay RG, Thompson EW. New insights on COX-2 in chronic inflammation driving breast cancer growth and metastasis. J Mammary Gland Biol. 2015; 20:109.

33. George OL, Ness SA. Situational awareness: Regulation of the myb transcription factor in differentiation, the cell cycle and oncogenesis. Cancers. $2014 ; 6: 2049-2071$
34. Arun $B$, Goss $\mathrm{P}$. The role of COX-2 inhibition in breast cancer treatment and prevention is. Semin Oncol. 2004; 31:22-29.

35. Singh B, Berry JA, Shoher A, Ramakrishnan V, Lucci A. COX-2 overexpression increases motility and invasion of breast cancer cells. Ann Surg Oncol. 2004; 11:S51.

36. Parida S, Mandal M. Inflammation induced by human papillomavirus in cervical cancer and its implication in prevention. European Journal of Cancer Prevention the Official Journal of the European Cancer Prevention Organisation. 2014; 23:432.

37. Huang OC, Huang RY. The cyclooxygenase-2/thromboxane A2 pathway: A bridge from rheumatoid arthritis to lung cancer? Cancer Lett. 2014; 354:28-32.

38. Aziz F, Qiu Y. The role of anti-LeY antibody in the downregulation of MAPKs/COX-2 pathway in gastric cancer. Curr Drug Targets. 2014; 15:469-476.

39. Tougeron D, Sha D, Manthravadi S, Sinicrope FA. Aspirin and colorectal cancer: Back to the Future. Clinical Cancer Research An Official Journal of the American Association for Cancer Research. 2014; 20:1087-1094.

40. Li Y, Niu Y, Sun Y, Mei L, Zhang B, Li Q, Liu L, Zhang R, Chen J, Mei Q. An apple oligogalactan potentiates the growth inhibitory effect of celecoxib on colorectal cancer. Nutrition \& Cancer-an International Journal. 2014; 66:29-37.

41. Kraus S, Sion D, Arber N. Can we select patients for colorectal cancer prevention with aspirin? Curr Pharm Design. 2015; 21:5127-5134

42. Chan AT, Ogino S, Fuchs CS. Aspirin and the risk of colorectal cancer in relation to the expression of COX-2 N Engl J Med. 2007: 356.2131-2142.

43. Bell D, Roberts D, Karpowicz M, Hanna EY, Weber RS, Elnaggar AK. Clinical significance of Myb protein and downstream target genes in salivary adenoid cystic carcinoma. Cancer Biol Ther. 2011; 12:569. 